\title{
A Liquid Cryogen Absorber for MICE*
}

\author{
D. E. Baynham ${ }^{1}$, P. Bish' ${ }^{2}$ T. W. Bradshaw ${ }^{1}$, M. A. Cummings ${ }^{3}$, \\ M. A. Green ${ }^{2}$, S. Ishimoto ${ }^{4}$, I. Ivaniouchenkov ${ }^{1}$, W. Lau, \\ S. Q. Yang ${ }^{5}$, and M. S. Zisman ${ }^{2}$
}

1. CCLRC Rutherford Appleton Laboratory ASTeC, Chilton-Didcot, OX11-0QX, UK

2. Lawrence Berkeley National Laboratory, Berkeley CA 94703, USA

3. Northern Illinois University, DeKalb IL 60011, USA

4. KEK Laboratory 1-1 Oho, Tsukuba, 305-0801, Japan

5. Oxford University Physics Department, Oxford OX1-3RH, UK

\section{August 2005}

Presented at the 2005 Cryogenic Engineering Conference Keystone Colorado, USA

29 August through 2 September 2005

Published in Advances in Cryogenic Engineering 51

\footnotetext{
* This work was supported by the Oxford University Physics Department and the Particle Physics and Astronomy Research Council of the United Kingdom. This work was also performed at the Lawrence Berkeley National Laboratory with the support of the Office of High Energy Physics, United States Department of Energy under DOE contract DE-AC0205CH11231. DOE funding for the US Neutrino Factory and Muon Collider Collaboration is gratefully acknowledged.
} 


\title{
A LIQUID CRYOGEN ABSORBER FOR MICE
}

\author{
D. E. Baynham ${ }^{1}$, P. Bish ${ }^{2}$, T. Bradshaw ${ }^{1}$, M. A. Cummings ${ }^{3}$, M. A. Green ${ }^{2}$, \\ S. Ishimoto ${ }^{4}$, I. Ivaniouchenkov ${ }^{1}$, W. Lau ${ }^{5}$, S. Q. Yang ${ }^{5}$, and M. S. Zisman ${ }^{2}$ \\ ${ }^{1}$ CCLRC Rutherford Appleton Laboratory ASTeC \\ Chilton-Didcot, OX11-0QX, UK \\ ${ }^{2}$ Lawrence Berkeley National Laboratory \\ Berkeley CA 94720, USA \\ ${ }^{3}$ Northern Illinois University \\ DeKalb IL 60011, USA \\ ${ }^{4}$ KEK Laboratory 1-1 Oho \\ Tsukuba, 305-0801, Japan \\ ${ }^{5}$ Oxford University Physics Department \\ Oxford OX1-3RH, UK
}

\begin{abstract}
The Muon Ionization Cooling Experiment (MICE) [1] will test ionization cooling of muons. In order to have effective ionization cooling, one must use an absorber that is made from a low- $z$ material. The most effective low $z$ materials for ionization cooling are hydrogen, helium, lithium hydride, lithium and beryllium, in that order. In order to measure the effect of material on cooling, several absorber materials must be used. This report describes a liquidhydrogen absorber that is within a pair of superconducting focusing solenoids. The absorber must also be suitable for use with liquid helium. The following absorber components are discussed in this report; the absorber body, its heat exchanger, the hydrogen system, and the hydrogen safety. Absorber cooling and the thin windows are not discussed here [2] [3].
\end{abstract}

KEYWORDS: Muon Ionization Cooling, $\mathrm{LH}_{2}$, LHe, and Hydrogen Safety

PACS: 07.20Mc, 14.60Ef, 25.30Mr, 27.10+h, 27.30+t 06.60Wa

\section{AN INTRODUCTION TO MUON IONIZATION COOLING}

Ionization cooling has been selected as a cooling method for a neutrino factory, because stochastic cooling, electron cooling and laser cooling take a long time $(>1 \mathrm{~s})$ compared to the lifetime of a muon (2.1 $\square \mathrm{s}$ at rest). When a muon enters a material, energy is lost along the track. This means that both longitudinal and transverse momenta are reduced as the muon passes through the material. If the muon is re-accelerated in the longitudinal direction, the reduction of transverse momentum is retained and beam cooling has been achieved. Multiple Coulomb scattering of the muon beam in the material counters the effect of cooling. If the emittance reduction is greater than scattering emittance growth, net ionization cooling results. 
TABLE 1. A Comparison of the properties of various ionization cooling absorber materials

\begin{tabular}{cccccc}
\hline Material & $\begin{array}{c}\mathrm{dE} / \mathrm{dx}^{-1} \\
\left(\mathrm{MeV} \mathrm{g}^{-1} \mathrm{~cm}^{2}\right)\end{array}$ & $\begin{array}{c}\mathrm{L}_{\mathrm{R}} \\
\left(\mathrm{g} \mathrm{cm}^{-2}\right)\end{array}$ & $\begin{array}{c}\text { Density } \\
\left(\mathrm{g} \mathrm{m}^{-3}\right)\end{array}$ & $\begin{array}{c}10 \mathrm{MeV} \text { Length } \\
(\mathrm{mm})\end{array}$ & $\begin{array}{c}\text { Equilibrium } \\
\text { Cooling Factor }\end{array}$ \\
\hline $\mathrm{LH}_{2}$ & 4.12 & 61.3 & 70.8 & 342.8 & 1.000 \\
$\mathrm{LHe}$ & 1.94 & 94.3 & 125 & 412.4 & 0.524 \\
$\mathrm{LiH}$ & 1.89 & 79.3 & $\sim 780$ & 67.8 & 0.352 \\
$\mathrm{Li}$ & 1.65 & 82.8 & 534 & 113.5 & 0.268 \\
$\mathrm{Be}$ & 1.61 & 85.2 & 1848 & 33.6 & 0.172 \\
$\mathrm{Al}$ & 1.62 & 24.3 & 2700 & 22.8 & $\sim 0.05$ \\
\hline
\end{tabular}

An equation that describes ionization cooling can be stated as follows [4]:

$$
\frac{d \square_{i, N}}{d z}=\square \frac{1}{\square^{2}} \frac{\square_{x, N}}{E} \boxminus d d z \square+\square_{\square} \frac{(0.014 G e V)^{2}}{2 \square^{3} E m_{\square} L_{R}}
$$

where $\square_{k, N}$ is the muon emittance; $\square=v / c ; E$ is the muon energy; $\square_{\square}$ is the transverse beam beta, $m$ is the mass of a muon and $L_{R}$ is the radiation length of the absorber material.

The term with the minus sign on the right hand side of Equation 1 is the cooling term; the term on the right hand side of Equation 1 with the plus sign is the coulomb scattering term. For effective ionization cooling one needs strong focusing in order to achieve a low value of $\square_{\square}$ and one wants to have a high value of $L_{R}$, which implies that the use of a low- $Z$ material for doing the cooling. In general, cooling is proportional to the number of electrons in the atom. Coulomb scattering is proportional to the number of charged nucleons in the atom squared. Thus hydrogen is the best material to use for ionization cooling. TABLE 1, which compares the properties of a number of liquid and solid absorbers, confirms this.

The $10-\mathrm{MeV}$ length shown in TABLE 1 is the material length needed to absorb $10 \mathrm{MeV}$ of energy from the muons. MICE intends to test absorbers of various types during the life of the experiment. When helium is used in place of hydrogen in the liquid absorber, the muon energy lost is $8.5 \mathrm{MeV}$ instead of $10.2 \mathrm{MeV}$ (over the $350 \mathrm{~mm}$ length). In order to reduce the muon energy $10.2 \mathrm{MeV}$ with beryllium, the plate thickness must be $34.3-\mathrm{mm}$. In order to test various materials in MICE, one must remove the liquid absorber and replace it with a solid absorber within a two week time period.

\section{THE ABSORBER FOCUS COIL MODULE}

The full MICE channel consists of two RF coupling coil modules (RFCC modules) [5] and three absorber focus coil modules (AFC modules). Intermediate configurations of the experiment will have reduced numbers of the RFCC modules and AFC modules. At the ends of the MICE cooling channel are tracker modules that are used measure the emittance of the muon beam entering the cooling channel and leaving the cooling channel. The change in emittance of the beam is a measure of muon cooling. FIGURE 1 shows a three-dimensional view of the full MICE cooling channel without the two tracker modules.

The AFC module consists of a pair of superconducting solenoids [6] mounted on a common aluminum mandrel, the magnet cryostat, and the absorber (liquid or solid) that is mounted at the center of the magnet. The vacuum around a liquid absorber is separate from the magnet vacuum or the MICE vacuum. The absorber 4-K cooler [2] and two 4-K magnet coolers [7] are also mounted on the top of the AFC module, which is between two RFCC modules or between an RFCC module and a tracker module (See FIG. 1). The two coils in the AFC module may be hooked up with opposite polarity (the flip mode) or with the same polarity (the non-flip mode). During the operation of the experiment, both modes of magnet operation will be used. FIGURE 2 shows a cross section of the AFC module. One can see the two-coil focusing magnet and the liquid cryogen absorber. During the course of the experiment, a solid absorber will sometimes replace the liquid absorber shown in FIG. 2. 


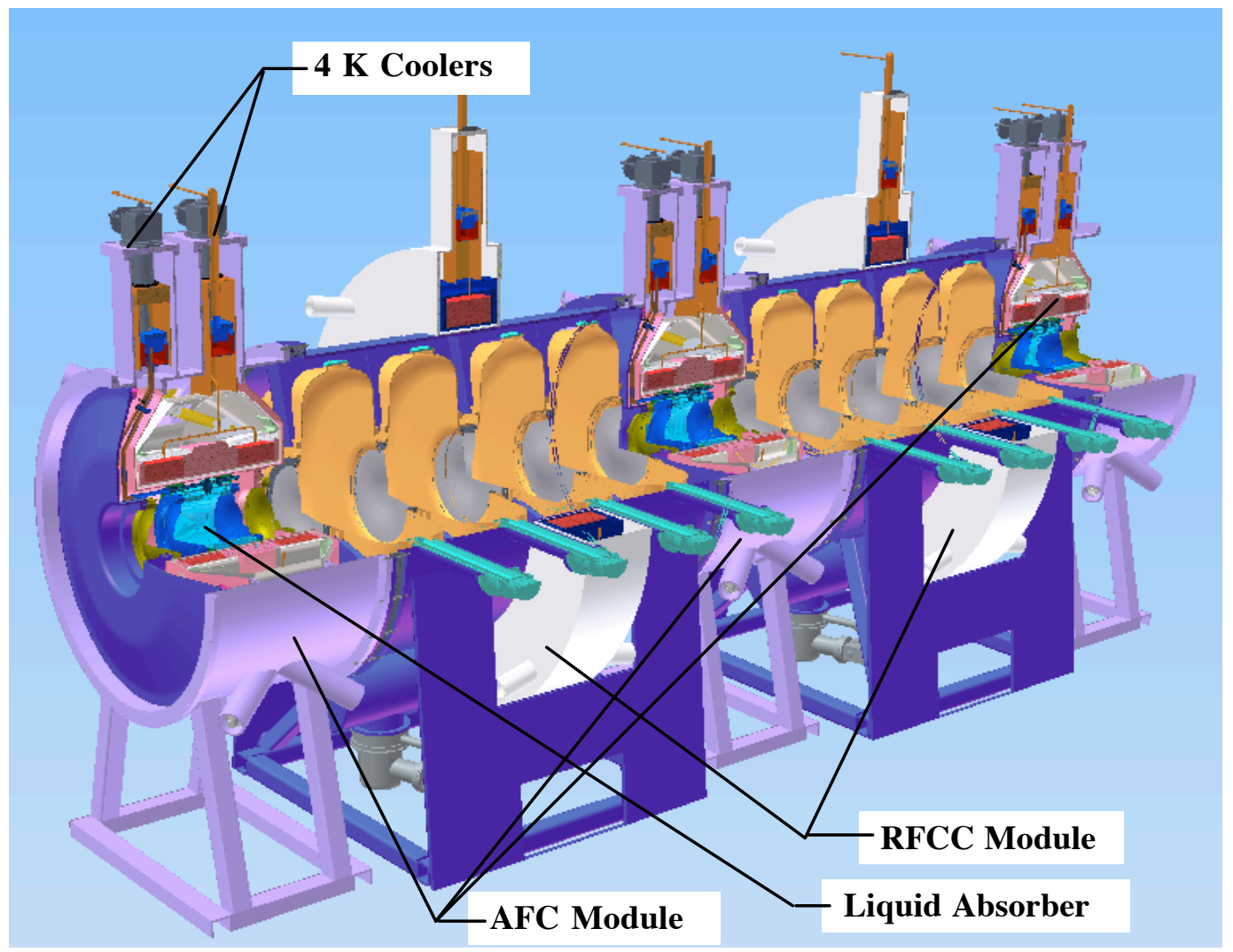

FIGURE 1. A three-dimensional view of the MICE cooling channel without the two tracker modules.

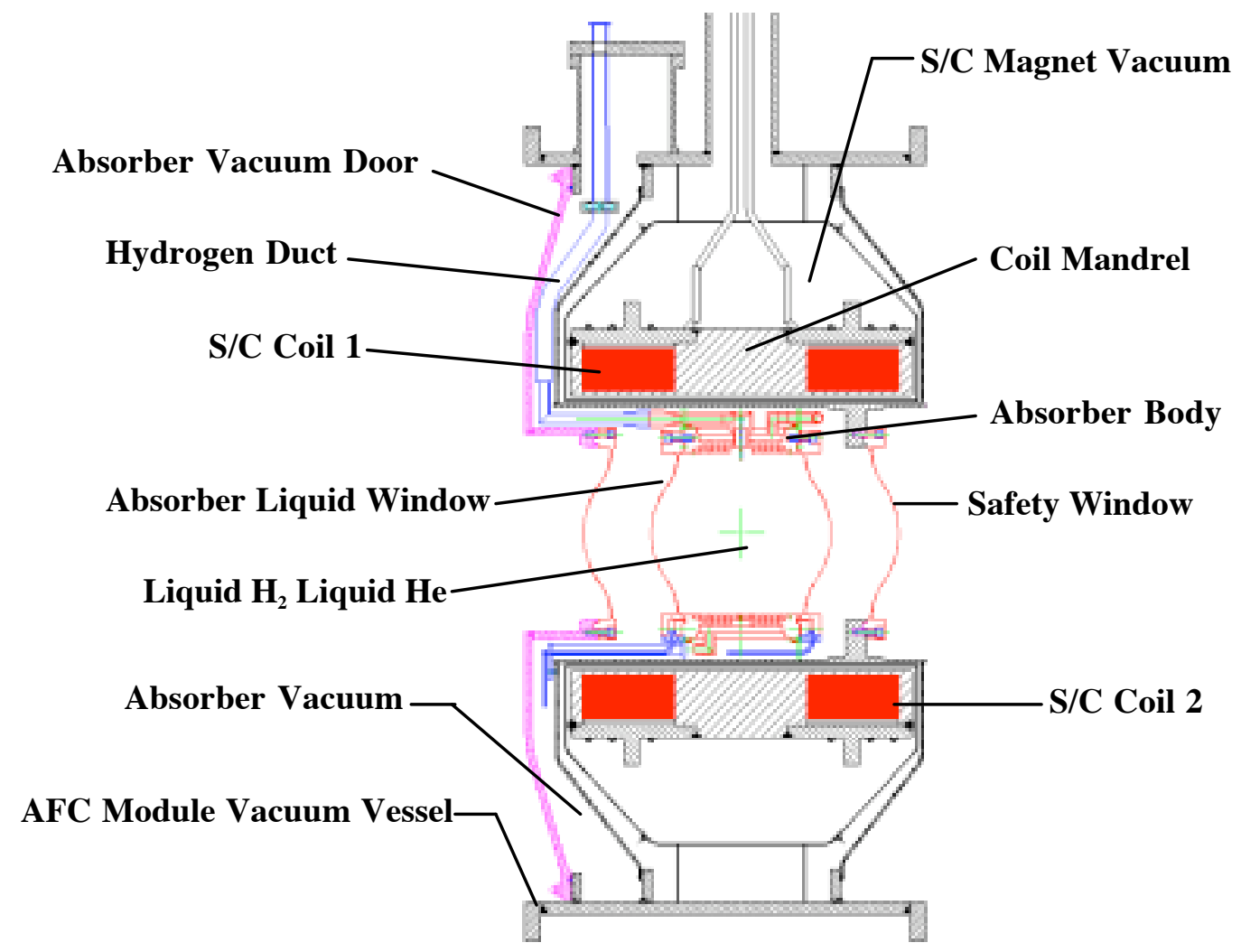

FIGURE 2. A cross-sectional view of the MICE absorber focus coil module showing the liquid absorber. 


\section{THE LIQUID CRYOGEN ABSORBER}

The operating requirements of MICE dictated the design of the liquid absorber. As an experiment, MICE is required to operate over a range of muon momenta, with a varying beam $\square_{\square}$ within the absorber. Since a range of absorber materials will be used over the life of the experiment, the liquid absorber must be easily removable. In order to change from liquid absorbers to solid absorbers, the absorbers must be warmed up so that AFC modules can be removed from the cooling channel. Once an AFC module has been removed from the cooling channel, the absorber vacuum door (with its thin safety window) is removed (see FIG. 2). The door removal permits disconnecting the hydrogen pipes between the cooler system and the absorber. The heat exchanger pipes (used for absorber cool down) are also disconnected, along with the instrumentation wires. The absorber, with its thin windows [3], is then removed as a unit from the warm bore of the magnet. Once the liquid absorber has been removed, it can be replaced by a solid absorber and the channel can be reassembled.

FIGURE 3 shows a cross-sectional view of the absorber within the warm bore of the superconducting focusing magnet. (Parts of the magnet are shown in FIG. 3.) The absorber shown in FIG. 3 consists of a 6061-T6 aluminum body with an extended heat-transfer surface on its inner cylindrical wall. The outside of the absorber body that faces into a cavity for liquid nitrogen or helium (used only for cooling the absorber down). The cavity side of the wall also has an extended surface. The heat exchanger built into the absorber is required for an absorber operating within a neutrino factory cooling-channel, but it is not required for the MICE absorber, because the muon beam intensity in MICE is low (no beam heating).

The 300-mm diameter thin-windows [3] (180- $\square \mathrm{m}$ thick at the window center) cover the ends of the absorber to form a closed volume that contains 20.4 liters of liquid hydrogen (or helium). The absorber volume is connected to the cooler and condenser through a liquid feed tube that goes into the bottom of the absorber. The second tube connected to the top of the absorber carries the boil-off gas (mixed with liquid) to a condenser attached to the cooler cold head [8]. Both of the tubes serve as vents in the event of a vacuum failure.

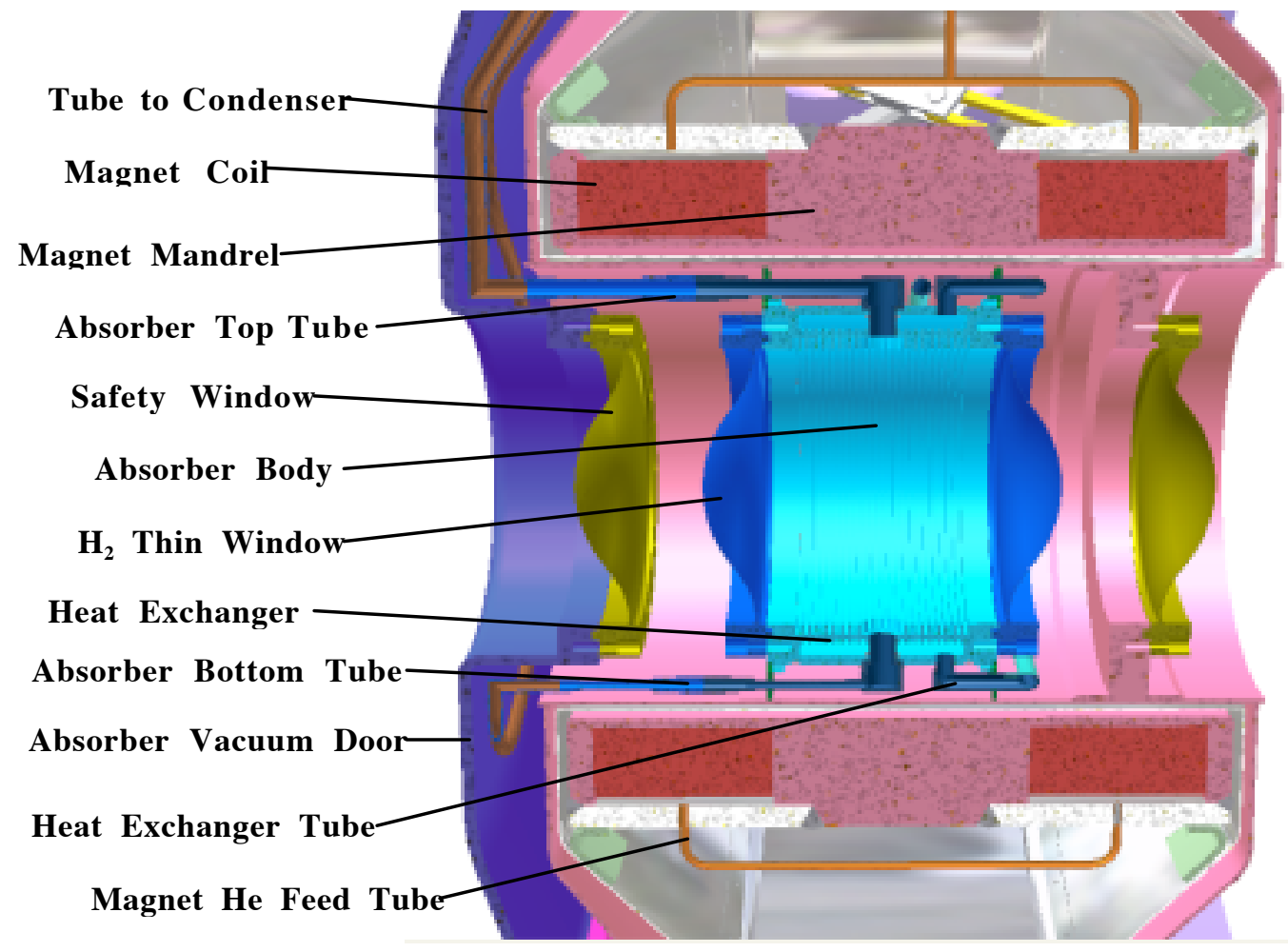

FIGURE 3. A three dimensional cross section of the MICE liquid absorber showing the absorber liquid volume, the absorber body, the body heat exchanger, the thin windows and the hydrogen feed tubes. 


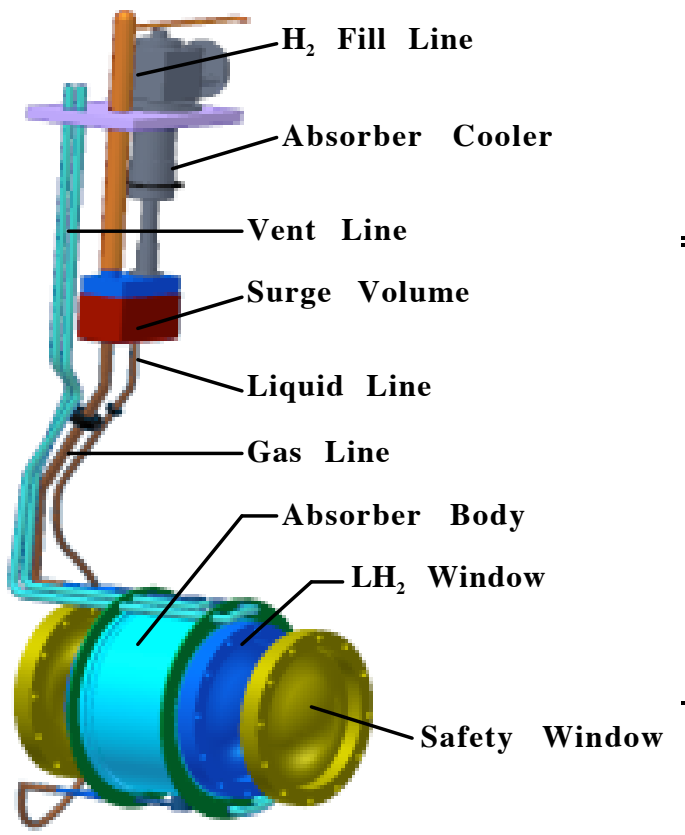

\section{Parameter}

\begin{tabular}{lc}
\hline \hline Absorber body inside diameter $(\mathrm{mm})$ & $\mathbf{3 0 0}$ \\
Absorber thin window diameter $(\mathrm{mm})$ & $\mathbf{3 0 0}$ \\
Length of the absorber $(\mathrm{mm})$ & $\mathbf{3 5 0}$ \\
Absorber volume @ 293 K (liters) & $\mathbf{2 0 . 7}$ \\
Length of the absorber feed tubes (m) & $\sim \mathbf{2 . 2}$ \\
Absorber feed tube ID (mm) & $\mathbf{> 1 5 . 0}$ \\
Surge tank volume (liters) & $\mathbf{2 . 0}$ \\
\hline
\end{tabular}

FIGURE 4. A three dimensional representation of the MICE liquid absorber, the condenser, and the $4 \mathrm{~K}$ cooler used to cool the absorber. The table within this figure presents the basic parameters of the MICE liquid absorber, the buffer volume and the system of pipes that connect the absorber to the cooler.

FIGURE 4 shows a three-dimensional representation of the MICE liquid absorber mounted within the AFC module. The demountable joints in the heat exchanger lines and the lines that connect the absorber volume to the condenser and surge volume will be in the space just below the condenser. Metal-seal demountable joints will be used to connect the absorber to the condenser-surge volume. The cooler and condenser-surge volume remains in the AFC module, when the liquid absorber is removed and replaced by a solid absorber. The table within FIG. 4 shows the basic parameters for the MICE liquid absorber. The dimensions and volumes shown apply at room temperature. At $20 \mathrm{~K}$, the volume of the absorber body is about 20.43 liters.

Not shown in FIG. 3 or FIG. 4 are the instrumentation and controls for the absorber and its cooling system. Monitoring of the absorber will be in the absorber body. The temperature of the absorber with liquid hydrogen or liquid helium within it will be used to control the liquid level in the absorber cryogenic system. A liquid level meter attached to the condensersurge volume will be used to turn the absorber heater on or off (depending on the liquid level). TABLE 2 shows operating parameters for the liquid absorber with bath liquid hydrogen or liquid helium within the absorber body. From TABLE 2, it is clear that operation with liquid helium determines the surge volume for the absorber system.

TABLE 2. Operating parameters of the MICE absorber operating with liquid helium and liquid hydrogen

\begin{tabular}{lcc}
\multicolumn{1}{c}{ Operating Parameter } & $\mathrm{LHe}$ & $\mathrm{LH}_{2}$ \\
\hline \hline Maximum absorber T at 0.17 MPa (K) & 4.82 & 22.1 \\
Cryogen triple point temperature (K) & $2.17^{*}$ & $13.8^{* *}$ \\
Absorber fill temperature (K) & 4.4 & $20.8^{* *}$ \\
Absorber fill pressure (MPa) & 0.12 & 0.12 \\
Maximum absorber operating T (K) & 4.6 & 21.0 \\
Minimum absorber operating T (K) & 3.8 & 15.0 \\
Liquid volume change over T range (liters) & 2.65 & 1.79 \\
\hline
\end{tabular}

* Triple point temperature for helium 4 is the lambda point temperature ** The temperatures given apply for hydrogen in the para state. 


\section{THE LIQUID ABSORBER HYDROGEN SYSTEM}

The MICE design team has chosen a commercial metal hydride bed as a source of hydrogen gas for the absorber. This choice was made because a system of large gas tanks (to contain the hydrogen in all of the absorbers) would be expensive and unwieldy. Using a hydride bed allows the liquid hydrogen in the absorber to be evaporated and returned to the hydride bed during a normal warm-up. The hydride bed will store more than $20 \mathrm{~m}^{3}$ of hydrogen at STP. The hydrogen is transferred from the hydride bed by heating the bed. Hydrogen is returned to the bed by cooling the bed with cold water from the chiller. The metal hydride bed is capable of taking up $\mathrm{H}_{2}$ gas at a rate of about $5 \mathrm{~m}^{3}$ per hour at STP.

Between the hydride bed and the liquid absorber is a $1-\mathrm{m}^{3}$ surge volume. This volume reduces the rate of pressure rise caused by a sudden air leak into the absorber vacuum space. When the absorber is being filled with hydrogen, the hydrogen from the hydride bed passes into the absorber where it is liquefied. Once the absorber is filled, the hydride bed is shut off from the gas buffer volume. Eventually the cooler cools the absorber and the hydrogen to the desired operating temperature. FIGURE 5 is a schematic representation for the MICE hydrogen system. Hydrogen lines are heavy; other gas lines are lighter. Not shown in FIGURE 5 is the absorber hydrogen zone boundary, which includes the hydride bed and the buffer volume. Hydrogen pipes between the buffer volume and the AFC module are jacketed with argon or $\mathrm{N}_{2}$ (if the pressure in the absorber is allowed to go below $0.11 \mathrm{MPa}$ ).

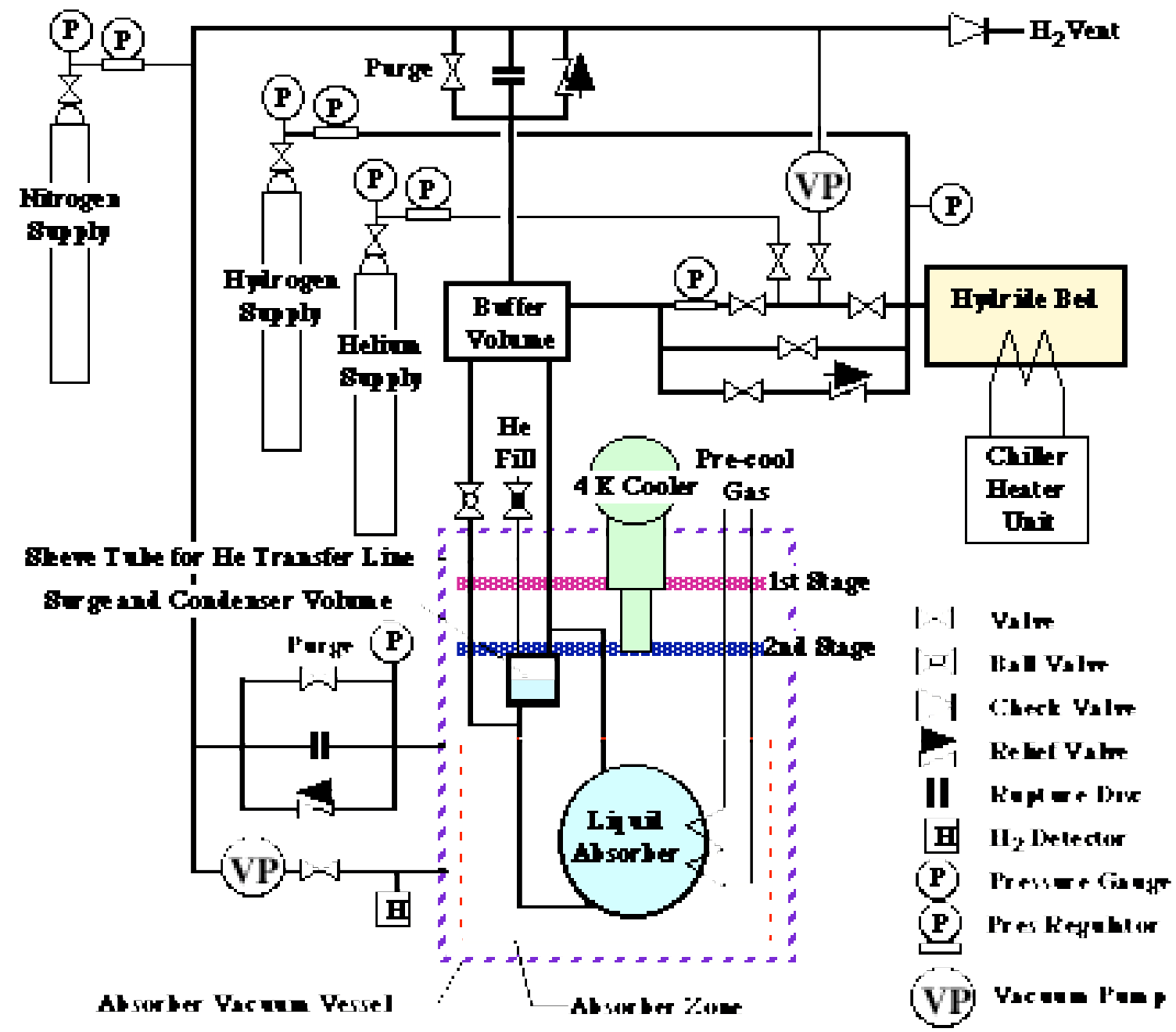

FIGURE 5. The MICE absorber hydrogen-system. This system includes the hydride bed, the external buffer tank, the absorber and its liquid surge tank, the absorber heat exchanger, and the emergency vent system. The box around the absorber denotes the absorber zone, which has four pipes that cross its boundary. These pipe have connectors that allow the absorber assembly to be removed from the AFC module. 
In FIG. 5, one can see that absorber boil-off gas goes directly to the external buffer volume through the two vents that connect directly to the absorber liquid volume. If the absorber vacuum vessel fills with liquid hydrogen (or helium), the boil-off gas is vented directly through the vacuum-vessel relief devices. If the pressure in the external buffer volume or the absorber vacuum vessel is above the design relief pressure (for the relief valve $\square \mathrm{P}=0.05 \mathrm{MPa}$ and for the burst disc $\square \mathrm{P}=0.09 \mathrm{MPa}$ for both the buffer tank and the absorber vacuum vessel), the gas is vented into the external emergency vent.

When the absorber is used as a helium absorber, the absorber is filled with liquid helium directly from an external tank. The line in FIG. 5 labeled He fill with a ball valve will allow a transfer line to enter the liquid buffer volume so that liquid helium can be fed directly into the bottom of the absorber. The helium gas is vented from absorber through the vent line from the top of the absorber. The absorber bottom vent is closed off during a helium fill.

\section{HYDROGEN SAFETY FOR THE MICE ABSORBER SYSTEM}

Hydrogen safety is a key design element for the MICE liquid absorber and its hydrogen supply system. Where possible, the design of the MICE system is based on well documented and well understood European and British safety standards. Because of the international character of the MICE collaboration, devices such as the absorbers themselves must be designed to Japanese and American safety standards as well. A hydrogen system test will be done at Rutherford Appleton Laboratory (RAL) as a test of all systems including the safety systems.

All of the components (cryostats, cryostat vacuum vessels, piping, buffer tanks, and the hydride bed) are designed in accordance with the applicable pressure vessel codes as applied to flammable liquids and gases. This means that the design safety margins are larger than those applied to the same vessels designed for non-flammable gases and liquids. The materials used for the hydrogen system must not be subject to hydrogen embrittlement.

The absorber windows and safety windows are designed in accordance with Fermilab safety standards for thin hydrogen windows, which require that the minimum working pressure be set to $0.17 \mathrm{MPa}(25 \mathrm{psi}$ ) and the minimum burst pressure (as determined by a number of windows being burst) must be greater than $0.68 \mathrm{MPa}(100 \mathrm{psi})$ at $293 \mathrm{~K}$ with the pressure inside the window. The window must withstand a $0.17 \mathrm{MPa}$ buckling external pressure. The buckling requirement allows for vacuum leak checking and an accidental pressurization of the absorber vacuum space, while the absorber itself is under vacuum.

The design of the relief valve system (or rupture disc) for the hydrogen system is based on the following conditions: 1) The absorber vacuum vessel is suddenly filled with air by an accident, while the absorber is filled with liquid helium [9]. (Because of its low heat of vaporization, liquid helium produces a much larger boil-off mass flow than liquid hydrogen.) 2) There are 10 layers of MLI on the absorber body, and it is assumed that there is no MLI on the thin windows. 3) The absorber body has two 15-mm ID vent paths from the top and bottom of the absorber to a point where the vent pipe connects to the first stage of the cooler. From the first stage of the cooler to the external buffer tank the vent pipes can be larger. This will allow the liquid absorber (with helium) to be vented with the peak pressure in the absorber less than $0.2 \mathrm{MPa}$, with a relief valve setting of $0.17 \mathrm{MPa}$.

The absorber vacuum vessel and its thin windows are also designed for a working pressure of $0.17 \mathrm{MPa}$. The absorber vacuum-vessel relief system is designed for the case when an absorber window ruptures, spilling over 20 liters of liquid hydrogen or liquid helium into the vacuum vessel [10]. A liquid helium spill into the vacuum vessel produces gas far more rapidly than a liquid hydrogen spill, so the relief system is sized for a helium spill.

Most of the absorber vacuum vessel is covered by another vacuum (either the MICE vacuum or the vacuum for the superconducting magnet). Only a small portion of the absorber vacuum is exposed to air. Since, the absorber and its cooler must be demountable from the AFC module and the absorber has thin hydrogen windows, oxygen buildup from small leaks in the absorber vacuum vessel are considered to be a potential safety hazard. A study of this hazard suggests that the issue may be important if the hydrogen window bursts, flooding the absorber vacuum with hydrogen. As result, the absorber vacuum vessel will be blanketed by a nitrogen atmosphere that is less than 1 percent oxygen [11]. 


\section{CONCLUDING COMMENTS}

The MICE liquid absorber is located at the center of each AFC module. The liquid absorber and the AFC module are designed so that the absorber can be removed and be replaced by a solid absorber (or another liquid absorber). The MICE liquid absorber and its cryogenic cooling system are designed so that the liquid absorber can be operated with either hydrogen or helium. The MICE liquid absorber will be kept cold using a single small $4 \mathrm{~K}$ cooler. The cooler is connected to the absorber through a gravity feed heat pipe that insures that the temperature difference between the fluid in the absorber and the cooler second stage cold head is minimized.

The hydrogen for the MICE liquid absorber is supplied from a metal hydride bed. The hydride bed stores up to $20 \mathrm{~m}^{3}$ of hydrogen at STP. The hydride bed not only supplies hydrogen to the absorber as it is liquefied, but it takes hydrogen gas back from the absorber when it is emptied after a hydrogen run. Sudden surges of hydrogen gas, from a fault, cannot be taken back into the hydride bed, so an emergency hydrogen vent system is a part of the absorber hydrogen system. Helium is fed into the absorber as a liquid after the absorber has been cooled to $20 \mathrm{~K}$ or below.

Hydrogen safety is a key part of the design of the MICE absorber system design. The MICE absorber has passed its first safety reviews. Further reviews will occur as the engineering design progresses. A hydrogen system with a liquid volume, a cooler, the surge volumes, and a hydride bed will be tested before the final liquid absorber system is built.

\section{ACKNOWLEDGEMENTS}

This work was supported by the Oxford University Physics Department and the Particle Physics and Astronomy Research Council of the United Kingdom. This work was also performed at the Lawrence Berkeley National Laboratory with the support of the Office of High Energy Physics, United States Department of Energy under DOE contract DE-AC0205CH11231. DOE funding for the US Neutrino Factory and Muon Collider Collaboration is gratefully acknowledged.

\section{REFERENCES}

1. Gregoire, G. Ryckewaert, G., Chevalier, L., et al, "MICE and International Muon Ionization Cooling Experiment Technical Reference Document," http://hep04.phys.itt.edu/cooldemo, (October 2004)

2. Baynham, E., Bradshaw, T., Green, M. A., et al, "Cooling of the MICE Liquid Absorber Using a Small Cooler," Advances in Cryogenic Engineering 51, AIP Press, Melville NY, (This publication), (2005)

3. Lau, W., Yang S. Q., and Green, M. A., "The Development of a 6061 Window for the MICE Liquid Absorber," Advances in Cryogenic Engineering 51, AIP Press, Melville NY, (This publication), (2005)

4. Kaplan, D. M., "MuSat and MICE Experimental Verification of Ionization Cooling Techniques," presented at the First International Neutrino Factory Summer Institute, Abingdon UK 29 June 2003

5. Li, D., Green, M. A., Virostek, S. P., et al, "Progress on the RF Coupling Coil Module Design for the MICE Cooling Channel," Proceedings of the Particle Accelerator Conference, Knoxville TN (May 2005)

6. Yang, S. Q., Green, M. A., Barr, G., et al, "The Mechanical and Thermal Design for the MICE Focusing Solenoid Magnet System," IEEE Transactions on Applied Superconductivity 15, No. 2, p 1259, (2005)

7. Green, M. A., "Cooling the MICE Magnets using Small Cryogenic Coolers," Oxford University Physics Department Engineering Note 10, MICE Note 109, http://hep04.phys.itt.edu/cooldemo, (Sept. 2004)

8. Green, M. A., "Integration of Liquid Cryogen Cooling and Cryo-coolers with Superconducting Electronic Systems," Superconducting Science and Technology, ISEC-2003 Special Issue, (2003)

9. Green, M. A., "Safety Issues for an Absorber that is designed for Liquid Helium Operation as well as Liquid Hydrogen Operation." Oxford University Physics Department Engineering Note 14, MICE Note 113, http://hep04.phys.itt.edu/cooldemo, (January 2005)

10. Yang, S. Q. and Green, M. A., "The Effect of a Hydrogen Spill inside of a 300 K Vacuum Vessel on MICE Absorber Hydrogen Safety," Oxford University Physics Department Engineering Note 1, MICE Note 100, http://hep04.phys.itt.edu/cooldemo, (March 2004)

11. Green, M. A., "Hydrogen Safety Issues Compared to Safety Issues with Methane and Propane," Advances in Cryogenic Engineering 51, AIP Press, Melville NY, (This publication), (2005) 


\section{DISCLAIMER}

This document was prepared as an account of work sponsored by the United States Government.

While this document is believed to contain correct information, neither the United States Government nor any agency thereof, nor The Regents of the University of California, nor any of their employees, makes any warranty, express or implied, or assumes any legal responsibility for the accuracy, completeness, or usefulness of any information, apparatus, product, or process disclosed, or represents that its use would not infringe privately owned rights. Reference herein to any specific commercial product, process, or service by its trade name, trademark, manufacturer, or otherwise, does not necessarily constitute or imply its endorsement, recommendation, or favoring by the United States Government or any agency thereof, or The Regents of the University of California. The views and opinions of authors expressed herein do not necessarily state or reflect those of the United States Government or any agency thereof, or The Regents of the University of California. 